\title{
Epigenomic profiling using microarrays
}

\author{
Bas van Steensel ${ }^{1}$ and Steven Henikoff ${ }^{2}$
}

BioTechniques 35:346-357 (August 2003)

\begin{abstract}
Genes occupy only a minor fraction of genomes such as ours; however, histone and nonhistone chromosomal proteins and methylated DNA bases are distributed over both genic and nongenic regions. These widespread "epigenomic" features can be mapped and characterized by alternative applications of the same microarray technologies that have been used for conventional transcriptional profiling. Here we describe diverse microarray-based strategies for profiling patterns of DNA methylation, DNA replication, DNA binding, and chromatin-associated proteins and histone modifications. The rapid progress that is being made in developing and applying epigenomic profiling methods and the increasing availability of microarrays mean that epigenomic profiling is likely to become a standard research tool for understanding chromatin structure and gene expression during development.
\end{abstract}

\section{INTRODUCTION}

In recent years, we have witnessed the widespread adoption of genomic technologies for addressing problems in gene regulation, such as the elucidation of genetic networks (1,2). Progress has been possible because comprehensive DNA sequence data sets for an organism can be exploited using microarrays, proteomics, and other emerging technologies to study transcription, protein synthesis, and protein interactions on a genome-wide scale. At the same time, we are becoming increasingly aware that gene regulation also depends on epigenetic information that is not encoded in the sequence of $\mathrm{A}, \mathrm{C}$, $\mathrm{G}$, and $\mathrm{T}$ nucleotides (3).

There are at least two distinct classes of epigenetic information that can be inherited with chromosomes. One class is DNA methylation, in which a nucleic acid base is modified by a DNA methyltransferase. In eukaryotes, DNA methylation generally occurs at the $\mathrm{C} 5$ position of cytosine, a reaction that is carried out by various members of a single family of enzymes. The other class of epigenetic information involves changes in chromatin proteins, most conspicuously in the modification of histone tails.
Processes have evolved to maintain epigenetic marks through multiple rounds of cell division and, in some cases, through germ lines (4). For example, methyl-CpG is maintained in animals by DNA methyltransferase 1 (Dnmt1) (5). Dnmt1 follows behind the replication fork and methylates the unmethylated $\mathrm{C}$ residue of each $\mathrm{CpG}$ that is base paired with methyl-CpG (6). The methylation specificity of Dnmt1 for only hemi-methylated DNA suffices to propagate the methylation mark through successive rounds of cell division.

Many eukaryotes, including favorite model organisms such as yeast, flies, and nematodes, lack maintenance DNA methylation yet still display epigenetic inheritance. The basis for epigenetic inheritance of this type has been elucidated by recent studies of core histones and their associated proteins. We have learned that the heritability of the silent state depends on methylation of the Nterminal tail of histone $\mathrm{H} 3$ at lysine-9 or lysine-27, whereas active chromatin correlates with a variety of alternative histone modifications and the replication-independent deposition of the $\mathrm{H} 3$ variant, H3.3 $(7,8)$. Methylated lysines on histone $\mathrm{H} 3$ serve as binding sites for chromodomain-containing proteins such as heterochromatin-associated protein 1 (HP1) and Polycomb, which are proteins that appear to stabilize the heritably silent state.

In organisms that display both DNA methylation and chromatin-based inheritance, there are interrelationships that are only now being uncovered. Whereas Dnmt1 maintains most CpG methylation, which may result in histone methylation (9), non-CpG methylation appears to be directed by methyllysine 9 of histone H3 $(10,11)$. The apparent irreversibility of methyl-lysine makes it likely that this histone modification lasts as long as the modified histone remains in the nucleosome (12). Reversible histone modifications, such as the acetylation of lysines and phosphorylation of serines, might alter chromatin inheritance transiently.

The molecular mechanism by which histone modifications are established and maintained is still unclear, although in some systems methylation of lysine 9 of histone $\mathrm{H} 3$ is targeted by small interfering RNAs (13) and in others by CpG methylation (14). These processes are likely to be facilitated by a variety of chromatin-remodeling complexes (15). Nucleosome replacement might provide a means of erasing epigenetic information, with active genes apparently undergoing continual replication-

${ }^{1}$ Netherlands Cancer Institute, Amsterdam, The Netherlands and 'Fred Hutchinson Cancer Research Center, Seattle, WA, USA 
independent nucleosome assembly (8).

The rapid progress that is being made to understand these diverse mechanisms of non-DNA chromosomal inheritance is rather analogous to the triumphant early days of molecular biology, which began with the discovery of the double helix, followed by the elucidation of the genetic code. A half-century later, we are beginning to understand some of the fundamentals of non-DNA chromosomal inheritance. Indeed, one envisions a broad "epigenetic code" that would maintain genes in active or silent states after they have been activated or repressed by sequence-specific DNAbinding factors (7).

Parallels between the genetic code and epigenetic information motivate the development of genome-wide technologies that can reveal epigenetic patterns in the same way that genomics can be used to elucidate genetic networks. Accordingly, microarray-based methods have been adapted to reveal localization patterns of DNA-binding proteins (16-18) and DNA methylation (19-22), sometimes referred to as "epigenomics" (23). Epigenomic methodology has also been applied to the discovery of genome-wide histone modification patterns $(24,25)$. Epigenomics promises not only to aid in our understanding of basic biological processes but also has the potential to be applied in a clinical setting. For example, in profiling cancer cells, epigenomic methods may reveal patterns of DNA methylation, histone modifications, or binding of nonhistone chromatin proteins that might be diagnostic of malignancy (26).

Here we review the state of the art in epigenomics using microarray-based approaches, where we anticipate rapid technological progress and exciting new insights into how genetic regulatory mechanisms are established and propagated on a genome-wide scale.

\section{DNA METHYLATION MAPPING}

5-Methylcytosine $\left({ }^{\mathrm{m} 5} \mathrm{C}\right)$ is present in most eukaryotic genomes, ranging from an almost undetectable level in Drosophila DNA (27) to a large fraction of cytosines in the genomes of plants such as maize (28). In mammals, methylation patterns are complex and change during development, with the large majority of methylated cytosines found at $\mathrm{CpG}$ dinucleotides (9). A vast literature describes methylation patterns in mammals and in model plants, where the occurrence of DNA methylation in promoter regions is generally accompanied by gene silencing. In mammals, ${ }^{\mathrm{m}} \mathrm{CpG}$ is bound by proteins with a methyl-binding domain; one of these, MeCP2, is known to recruit a histone deacetylase (29) and a histone methyltransferase (30). In humans, the loss of methylation or loss of MeCP2 can lead to disease, including Immunodeficiency Craniofacial Syndrome and Rett Syndrome (31). Furthermore, abnormal DNA methylation is a hallmark of cancer cells, and epigenetic silencing of tumor suppressor genes is thought to be a causal basis for a large proportion of sporadic human cancers (32). Therefore, there is a growing interest in applying modern genomic technologies to study DNA methylation in healthy and diseased states.

Several different strategies have been applied to detect methylated bases (33). Complete digestion of DNA, followed by chromatography, electrophoresis, or mass spectrometry can be used to quantify methylation genomewide, and anti-m5 $\mathrm{C}$ antibody can be used to localize methylation patterns along chromosomes in situ. However, to determine methylation patterns along a DNA sequence, two basic strategies have been the most popular. One is to use methyl-sensitive restriction endonucleases to map and quantitatively assay the relative abundance of methyl-C at particular residues, and there are several variations of this basic approach (33). For example, MspI cleaves at $\mathrm{C}-{ }^{\mathrm{m}}{ }^{5} \mathrm{C}-\mathrm{G}-\mathrm{G}$, but HpaII cleaves only unmethylated C-C-G-G, and so simply by cleaving with two enzymes and quantifying bands on Southern blots of agarose electrophoretic gels, it is easy to determine the degree of $\mathrm{CpG}$ methylation at any particular CCGG sequence. However, it is often desirable to assay methylation at multiple sites along a sequence in a way that is not limited to any particular sequence motif. For this purpose, bisulfite sequencing is performed (34); cytosines are converted to uracils by the treatment of DNA with sodium bisul- fite, a procedure that leaves ${ }^{\mathrm{m} 5} \mathrm{C}$ unaffected. When a DNA polymerase encounters a uracil, it will insert an A residue into the complementary strand. As a result, all nonmethylated $\mathrm{C}$ residues will be mutated to $\mathrm{T}$ after PCR amplification. By cloning and sequencing a sample of products from an amplification reaction, the ratio of methylated to unmethylated bases can be determined at every cytosine in an amplified sequence segment.

These popular methods are limited to the determination of methylation patterns at individual loci. However, genomic profiling strategies are more challenging and have mostly been applied to $\mathrm{CpG}$ islands, which are dense clusters of unmethylated $\mathrm{CpG}$ dinucleotides that are frequently found at promoters (28). CpG islands are sometimes methylated in cancers, and so $\mathrm{CpG}$ methylation profiling of promoter regions might be used diagnostically. Differentially methylated $\mathrm{CpG}$ islands have been identified by differential cleavage using methylation-sensitive restriction endonucleases, ligation of end adapters, and PCR amplification (35). In an additional approach to isolate methylated $\mathrm{CpG}$ islands, restriction fragments containing $\mathrm{CpG}$ islands were first enriched, based on their high melting temperature, and then the methylated fraction was further enriched using a methyl-DNA-binding column (36).

Microarray analysis has been applied to the determination of methylation patterns on a genomic scale. One approach (Figure 1A) uses ligation-mediated PCR to preferentially amplify strongly methylated genomic regions (19). The amplification of nonmethylated regions is suppressed by the digestion of the template DNA before PCR with the restriction enzymes Bst $\mathrm{UI}$ and HpaII, which are blocked by cytosine methylation. This method was used to compare the methylation patterns from tumor and normal tissue samples by hybridizing to microarrays containing 7776 randomly cloned genomic DNA fragments that were enriched in $\mathrm{CpG}$ islands. Tumors could be classified based on the resulting methylation profiles, and specific $\mathrm{CpG}$ islands were identified that may serve as epigenetic tumor markers $(19,37)$. 
A related method (Figure 1B) (22) uses digestion with $S m a \mathrm{I}$, which cleaves the unmethylated sequence 5'-CCCGGG-3', followed by digestion with $X m a \mathrm{I}$, which is a methyl-insensitive isoschizomer of SmaI. The cleavage products of $X m a \mathrm{I}$ have $5^{\prime}$ protruding ends, whereas SmaI leaves blunt ends. Next, adaptors specific for the XmaI ends are ligated, and PCR is performed using primers that are complementary to these adaptors. The resulting PCR products, which, in principle, should contain only genomic regions with methylated 5'-CCCGGG-3' sequences, are hybridized to microarrays (22).

In another approach (Figure 1C), size fractionation after cleavage with a methyl-sensitive restriction enzyme leads to enrichment or depletion of fragments in which methylation blocks specific sites. This strategy was used to identify targets of the Arabidopsis CHROMOMETHYLASE3 (CMT3) DNA methyltransferase (20). In this study, genomic DNA from $\mathrm{cmt}^{-/-}$mutant plants that are deficient in $\mathrm{CpNpG}$ methylation (38) was digested with MspI, which cleaves C-C-G-G but does not cleave ${ }^{\mathrm{m} 5} \mathrm{C}-\mathrm{C}-\mathrm{G}-\mathrm{G}$. The low molecular weight fraction was labeled with a $\mathrm{Cy}^{\mathrm{TM}} 5$ dye, and the corresponding fraction from wild-type plants was labeled with a $\mathrm{Cy} 3$ dye, and the mixture was hybridized to microarrays. Because methylated DNA is depleted from the low molecular weight fraction, target loci could be identified as those with a high Cy5:Cy3 ratio. Using a small microarray of spotted PCR amplification products that primarily represented randomly chosen locations from the $\mathrm{Ara}$ bidopsis genome, we found that CMT3 preferentially targeted transposons, which is consistent with a role for $\mathrm{Cp}$ $\mathrm{NpG}$ methylation in genome defense.

Genomic assays have also been performed using a bisulfite-based strategy (Figure 1D). In this case, oligonucleotide pairs that differ by having either a $\mathrm{C}$ or a $\mathrm{T}$ at a methylatable position are present on an array, and discrimination is achieved by incubating at a temperature that allows only exact matches between the probe and the correct oligonucleotide to anneal $(39,40)$. This strategy was used to compare tumor versus nontumor tissues by assaying for the degree of methylation at selected $\mathrm{CpG}$ sites in genes that were thought to be involved in tumorigenesis. Using a machine learning algorithm to analyze the data, Adorjan and coworkers (40) found methylation profiling at 232 selected loci to successfully distinguish cancerous from control tissue and to distinguish between tumor types.

The different microarray-based strategies that have been used for methylation profiling have distinct features and provide different types of information (Table 1). Endonuclease cleavage combined with either size fractionation or PCR amplification typically has a mapping resolution of a few kilobases, without providing specific information about any individual methylated base. In contrast, the bisulfite-based strategy, which has a single base pair resolution, provides only information about specific residues that have been chosen in advance as being informative. Therefore, cleavage-based methods are suitable for surveying gene-sized targets, whereas the bisulfite-based procedure may be used when particular sites have been identified and oligonucleotide pairs optimized. Importantly, the restriction endonucleasebased approaches do not provide absolute values for the methylation levels of probed loci but only relative methylation levels in comparison to a co-hybridized reference sample. In contrast, the bisulfite-based approach allows for absolute measurements of methylation levels (after calibration) and does not involve co-hybridization of a reference sample $(39,40)$. We anticipate that improvements in microarray technologies and the availability of "tiled" arrays covering long contiguous regions will lead to improvements and a broader application of these and possibly other methylation profiling strategies.

\section{MAPPING OF DNA REPLICATION}

The timing of DNA replication of individual genomic sequences during S-phase is tightly regulated. Replication timing appears to be related to several other epigenetic phenomena, such as chromatin structure and transcriptional activity, and may function as an epigenetic marking mechanism (41). Two studies in eukaryotes have employed microarray-based techniques to generate genomic maps of replication timing. One study in yeast (42) performed differential labeling of unreplicated and replicated DNA with heavy

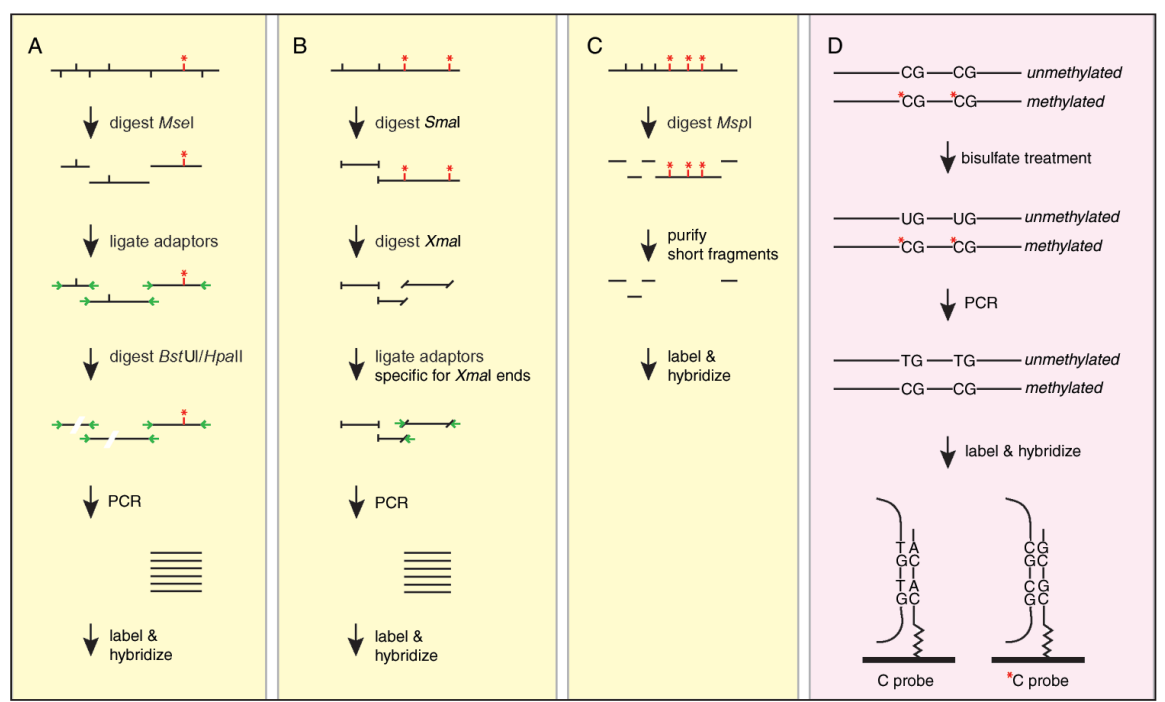

Figure 1. Methods for ${ }^{\mathbf{m} 5} \mathbf{C}$ profiling. (A-C) Restriction enzyme-based strategies for the selection of methylated (A and B) or unmethylated (C) genomic sequences. In each case, the same protocol is applied in parallel to both an experimental and a reference DNA sample, and hybridizations are performed as two-color co-hybridizations. Different ${ }^{\mathrm{m} 5} \mathrm{C}$-sensitive restriction enzymes may in principle be used in the procedures shown in panels A and C. (D) The strategy for the bisulfite detection of methylation patterns. For each sequence, the microarray contains two oligonucleotide probes; one to detect the unmodified sequence and another to detect the bisulfite-altered sequence. ${ }^{\mathrm{m} 5} \mathrm{C}$ is marked by an asterisk $\left(^{*}\right)$ and shown in red; adaptors for PCR amplification are indicated by short green arrows. 
Table 1. Comparison of Methods for Microarray-Based Detection of Cytosine Methylation

\begin{tabular}{|c|c|c|c|}
\hline & $\begin{array}{c}\text { Sodium Bisulfite } \\
\text { and PCR }\end{array}$ & $\begin{array}{c}\text { Restriction Digest } \\
\text { and Size Fractionation }\end{array}$ & $\begin{array}{l}\text { Restriction Digest } \\
\text { and PCR }\end{array}$ \\
\hline References & $(39,40)$ & (19) & $(18,21,37)$ \\
\hline $\begin{array}{l}\text { Sequences that } \\
\text { can be probed }\end{array}$ & In principle, any sequences. & $\begin{array}{l}\text { Limited to loci with pairs of } \\
\text { probed restriction sites. }\end{array}$ & $\begin{array}{l}\text { Limited to loci with pairs of probed } \\
\text { restriction sites that are close } \\
\text { together. }\end{array}$ \\
\hline $\begin{array}{l}\text { Amount of genomic } \\
\text { DNA used }\end{array}$ & $>10$ nga & $20-30 \mu \mathrm{g}$ & $1-2 \mu \mathrm{g}$ \\
\hline Type of microarray & $\begin{array}{l}\text { Specially designed paired } \\
\text { oligonucleotide arrays. }\end{array}$ & $\begin{array}{l}\text { Any microarray with regions } \\
\text { of interest. }\end{array}$ & $\begin{array}{l}\text { Any microarray with regions } \\
\text { of interest. }\end{array}$ \\
\hline Resolution & Single nucleotide. & $0.5-3 \mathrm{~kb}$ & $0.5-3 \mathrm{~kb}$ \\
\hline Current application & Human & Arabidopsis genomic sequences. & Human CpG islands. \\
\hline
\end{tabular}

and light isotopes. Microarray hybridizations of light and heavy DNA sampled at various times after the start of S-phase allowed for the determination of the timing of replication of the entire yeast gene complement. In another experimental approach using cultured Drosophila cells (43), nascent DNA was labeled with 5-bromo-2-deoxyuridine (BrdU) and purified from cells in either early or late S-phase. Comparative hybridization of the nascent DNA samples to cDNA arrays yielded a detailed genomic map of replication timing. These experiments revealed an intriguing difference between yeast and Drosophila; a clear correlation was observed between replication timing and transcriptional activity of genes in Drosophila (43), but no such correlation was found in yeast (42).

\section{MAPPING OF PROTEIN-DNA INTERACTIONS: CHROMATIN IMMUNOPRECIPITATION}

Chromatin immunoprecipitation (ChIP) has become an important tool for studies of chromatin and proteinDNA interactions in vivo. Most mapping of histone modifications is derived from ChIP experiments. In addition, ChIP has been instrumental in revealing many protein-DNA interactions in the living cell. The technique is usually based on in vivo cross-linking of protein-DNA complexes by chemical agents or ultraviolet light (Figure 2A). The cross-linked chromatin is subsequently subjected to mechanical shearing to obtain small DNA fragments, after which specific protein-DNA complexes are purified by immunoprecipitation with an antibody against a chromatin protein or histone modification of interest. Finally, the cross-links are reversed, and the co-immunoprecipitated DNA is analyzed for the presence of specific genomic sequences (44). The combination of ChIP with microarray technology (ChIP-on-chip) has allowed for the parallel detection of protein binding to thousands of loci, facilitating the construction of whole-genome maps of histone modifications and binding patterns of DNA-associated proteins.

It is useful to consider the underlying chemistry of ChIP. Formaldehyde is by far the most frequently used crosslinker in ChIP experiments. In principle, formaldehyde can create both protein-protein and protein-DNA cross-links (44). However, in the paper that described the cross-linking protocol and laid the foundation for today's ChIP technology, Solomon and Varshavsky (45) reported that in vitro formaldehyde could efficiently crosslink histones to nucleosomal DNA but failed to cross-link two DNA-binding factors ( $\alpha$ factor and the lac repressor) to their cognate-binding sequences. This suggested that the cross-linking efficiency of formaldehyde could vary greatly between different protein-DNA complexes. Given the fact that histones are rich in basic amino acid residues (the primary target of formaldehyde) and are tightly wrapped with $147 \mathrm{bp}$ DNA, it may not be surprising that the cross-linking of DNA to nucleosomes is very robust.

For nonhistone proteins, ChIP has become more successful than one may have predicted from the initial report (45), particularly in yeast. Wholegenome maps have been reported for a large number of yeast transcription factors $(16,17,46,47)$. It is possible that many DNA-binding proteins are in fact not directly cross-linked to DNA when formaldehyde is applied in vivo, but rather to nearby nucleosomes because formaldehyde is an efficient proteinprotein cross-linker. From a practical point of view, it might not often matter whether cross-linking occurs directly to DNA or indirectly via nucleosomes. However, one may ask whether efficient cross-linking of a given DNAbinding factor depends on the precise position of the factor relative to the nearest nucleosome rather than on binding to DNA per se. Thus, locusspecific differences in "cross-linkability" may, in some cases, bias the results of ChIP experiments. Moreover, certain antibody epitopes might be sterically blocked, depending on the complex that a protein is part of, which may also create a bias in the detected binding pattern. Unfortunately, such effects can sometimes be difficult to rule out experimentally.

The mapping resolution of ChIP is 
primarily determined by the size of the chromatin fragments used for the immunoprecipitation. In various ChIP protocols, chromatin is sheared into fragments, with an average size ranging from 400 to $3000 \mathrm{bp}(25,46-48)$. The mapping resolution of ChIP may be expected to be in this range. Thus, with the current ChIP protocols, microarrays with probes spaced from 500 to 1000 bp provide sufficient density to monitor protein-binding events in a genomic region of interest. Note that the mapping resolution of ChIP may not be uniform throughout the genome; it is possible that the efficacy of mechanical shearing depends on the chromatin conformation or the degree of compaction, which might differ between loci. The mapping resolution of ChIP might be further improved by increased fragmentation (e.g., by nuclease digestion).

ChIP-on-chip experiments have been particularly successful in Saccharomyces cerevisiae. The relatively small size and low complexity of the yeast genome has allowed for the construction of true whole-genome arrays, including all transcription units and all intergenic regions $(16,17,25,46,47)$. For example, Lieb and coworkers (46) used whole-genome arrays that consisted of about 13,000 spotted PCR products, representing genomic fragments with median sizes of approximately $1100 \mathrm{bp}$ for transcribed regions and 370 bp for intergenic regions. Primer sets for the construction of these arrays have become commercially available.

Several groups have succeeded in obtaining binding maps of DNA-associated proteins in yeast. In all cases, the same basic protocol was followed, with only minor modifications. Whole-genome maps have been reported for histone modifications $(24,49)$, chromatin-modifying proteins $(25)$, and DNA-binding factors $(16,17$, 46,47). In an impressive effort, Lee and colleagues (47) have performed systematic mapping of the genomic binding sites of virtually all known transcription factors in budding yeast. Of 141 factors included in their study, they managed to express epitopetagged versions of 106, and used these for whole-genome location studies using ChIP and hybridizations on microarrays carrying all yeast intergenic regions. Specific binding patterns were observed for the majority of these factors, which were generally in good agreement with data from previous literature.

Despite these successes in yeast, the application of ChIP-on-chip in higher eukaryotes faces additional challenges. The tremendous size and complexity of the genomes of multicellular model organisms make it more difficult and prohibitively expensive for most laboratories to construct genomic microarrays that encompass even a single chromosome. Furthermore, in larger genomes, it is more difficult to probe individual loci without being hampered by background signals due to the cross-hybridization of related sequences. In addition, chromatin composition is more complex in higher eukaryotes. For example, Drosophila melanogaster has about 5-fold more genes encoding transcription factors than yeast and, in mammals, this number is about 15 fold. A large number of splicing variants may raise this number even further. Because of the higher complexity of chromatin in multicellular organisms, antibodies used for the immunoprecipitations must have excellent specificity. Finally, in multicellular organisms, it may be difficult to achieve the required rapid penetration of crosslinking reagent into tissues and, once cross-linked, it may be problematic to isolate intact chromatin from tissue.

Despite these technical challenges, several reports suggest that ChIP-onchip in mammalian cells is feasible for at least some proteins. Weinmann, Wells, and coworkers $(48,50)$ have used their microarray of 7776 cloned $\mathrm{CpG}$ islands in ChIP-on-chip experiments to successfully identify target loci of human $\mathrm{E} 2 \mathrm{~F}$ and $\mathrm{Rb}$ proteins in cultured cells. A large fraction of these targets were confirmed by conventional ChIP, which suggests the high reliability of the microarray detection. Ren et al. (51) constructed a microarray of 1444 human proximal promoter regions and applied ChIP-on-chip technology and a robust statistical model to identify 127 in vivo targets for E2F4. Efforts are under way to construct larger promoter arrays and even wholechromosome arrays, and undoubtedly these will be used to generate increas- ingly more detailed binding maps of histone modifications and DNA-binding factors.

\section{MAPPING OF PROTEIN-DNA INTERACTIONS BY TARGETED ADENINE METHYLATION}

A different technique for the detection of protein-DNA interactions uses the marking of protein-binding sites by DNA adenine-methyltransferase (dam) $(18,52)$. This technique, named dam identification (DamID), does not involve cross-linking or require antibodies or purification of protein-DNA complexes. Instead, a fusion protein consisting of Escherichia coli dam and a chromatin protein or transcription factor of interest is expressed in cultured cells or in a multicellular model organism such as Drosophila (Figure 2B). As a consequence, the tethered dam is targeted to the native genomic binding sites of its fusion partner, which leads to the preferential methylation of adenines close to these binding sites. Adenine methylation does not occur endogenously in the DNA of animals and fungi. Thus, methylation by dam provides a unique tagging system to mark the genomic binding sites of the chromatin protein.

After a sufficient time span of expression of the dam fusion protein to allow for the accumulation of the methylation tags, genomic DNA is isolated, and the adenine methylation patterns are mapped. Several methods are available for the detection of adenine methylation; these methods typically rely on restriction enzymes that are sensitive to adenine methylation. Similar to ${ }^{5} \mathrm{C}$, adenine methylation levels of individual loci can be determined by Southern blot analysis or quantitative PCR methods. There is no equivalent to bisulfite sequencing for adenine methylation. In the most powerful application, the mapping of adenine methylation patterns is performed using DNA microarrays, which allows for the screening of thousands of loci at once (18).

$E$. coli dam is a single polypeptide of $32 \mathrm{kDa}$ that retains its methyltransferase activity when fused to other proteins either at its $\mathrm{C}$ - or N-terminus (52). Dam methylates the 6-position of adenine in the DNA sequence GATC. Ade- 
nine methylation is an almost ideal tag to mark genomic sequences in vivo. It is a small modification that has little effect on overall DNA topology, although it somewhat reduces helix stability and could in theory affect protein interactions with the major groove (53). Both in S. cerevisiae and in D. melanogaster, the expression of dam does not appear to have any detectable effect on growth, fertility, or viability (54-57). High levels of adenine methylation (up to $50 \%$ of all GATC sequences) have no detectable effect on fly development (56), which is a very sensitive indicator of defects in cell-cycle regulation and gene expression. No data are currently available on the toxicity of dam in mammalian cells, but the expression of PaeR7 adenine methyltransferase in a mouse cell line did not have any detectable effect on cell growth (58).

Because of the high enzymatic activity of dam, only a trace amount of the dam protein needs to be expressed in vivo. In fact, higher expression leads to saturating levels of methylation (both targeted and nontargeted) in the genome, which would obviously corrupt the mapping of protein target sites. Optimal expression levels are so low that we have been unable to detect the dam fusion protein by immunofluorescence microscopy or Western blot analysis (52). This low expression level makes it unlikely that the fusion protein interferes with the functions of the endogenous chromatin protein or its targets. Despite the low intracellular concentration, the fusion protein appears to be readily able to compete with the typically much higher concentrations of endogenous protein for binding sites. This may be explained by the extremely dynamic behavior of chromatin proteins in the nucleus; photobleaching experiments have shown that virtually all chromatin-associated proteins rapidly exchange between the genome-bound and free-diffusing states (59), usually within a few minutes. Thus, the exposure of cells to a dam fusion protein for several hours will allow the fusion protein molecules to visit and methylate most of the target loci.

Experiments in flies and in budding yeast have shown that methylation by tethered dam spreads in cis over $5 \mathrm{~kb}$ from the genomic binding site $(52,57)$, with the most methylation occurring in Drosophila within a 2-kb range (52). Most likely, the high flexibility and mobility of chromatin in the living cell causes the tethered dam to "bump" into adjacent sequences. In support of this interpretation, tethered dam has been found to carry out methylation in trans when a genomic region is spatially positioned close to the binding site of the dam fusion protein (57). In vitro, the dam displays linear diffusion (tracking) over several kilobases along the DNA double helix (60), which may also contribute to the cis spreading of methylation in vivo. Regardless of the precise mechanism, the spreading has impor- tant practical implications. On the one hand, it allows for the probing of almost every region in the genome for interaction with a protein of interest because GATC sequences occur on average once every 200-300 bp. On the other hand, the mapping resolution of DamID is limited by the range of spreading. Fortunately, computational approaches can often identify putative consensus binding sequences among the identified targets, thus effectively increasing the mapping resolution (61). If DNA tracking by dam indeed contributes to the spreading of methylation, then it might be possible to increase the mapping resolution by employing a dam enzyme lacking this tracking ability (60).

To map adenine methylation patterns by means of DNA microarrays, methylated regions are purified from genomic DNA, labeled with a fluorescent dye, and hybridized to a microarray that carries sequences of interest. The intensity of the fluorescent signal in each spot on the microarray is pro-



Figure 2. Methods for profiling of in vivo protein-DNA interactions. (A) The principle of chromatin immunoprecipitation (ChIP). The control sample may either be derived from total chromatin $(16,47)$ or from a mock immunoprecipitation $(15,24,46)$. (B) The principle of DNA adenine-methyltransferase (dam) identification (DamID). Dam is indicated by a pink oval, and the dam-fusion protein is indicated by a pink oval linked to a red oval. Methylated DNA is marked in blue. 
portional to the abundance of the probed sequence in the methylated DNA fraction. The purification of methylated regions is done by the digestion of genomic DNA with DpnI, which cuts Gm6ATC but not GATC sequences, followed by size fractionation to obtain small fragments (somewhat arbitrarily chosen to be $<2.5 \mathrm{~kb}$ ) that are highly enriched for methylated regions (18). A disadvantage of this approach is that large amounts of genomic DNA (about $1 \mathrm{mg}$ ) are required to obtain sufficient amounts of methylated fragments for microarray hybridization. However, we have recent6y developed an amplification protocol that allows for approximately 1000-fold lower amounts of starting material to be used (F. Greil and B.v.S., unpublished data).

For detection using microarrays, two-color hybridizations are typically performed. In the case of DamID, the "experimental" sample of methylated DNA fragments from cells expressing the dam fusion protein is labeled with one fluorescent dye and co-hybridized with a "reference" sample consisting of methylated genomic regions purified from control cells that express unfused dam, labeled with a different fluorescent dye. Target loci of the protein of interest are identified by their high experimental:reference fluorescence ratio (i.e., dam fusion:dam only) as compared to nontarget loci. This direct comparison of the methylation levels obtained with a dam fusion protein to those obtained with dam alone controls for chromatin accessibility and other differences, which ensures that the loci identified are those at which methylation levels are elevated due to the binding of the chromatin protein of interest (18).

DamID is still a relatively new method and has only been reported in Drosophila to date. Targeted methylation also works in yeast (57) but has not yet been used to identify novel targets of chromatin proteins. In Drosophila, mapping studies have been reported for sequence-specific DNA-binding factors, including GAGA factor $(18,61)$ and members of the Max family of regulatory proteins (62). In each case, the protein-binding patterns correlated with the occurrence of the known consensusbinding motifs of these proteins, which

Table 2. Comparison of Methods for Microarray-Based Detection of In Vivo Protein-DNA Interactions

\begin{tabular}{|c|c|c|}
\hline & ChIP-on-Chip & DamID \\
\hline References & $(15,16,23,24,46-51)$ & $(17,61,62)$ \\
\hline Antibody required & Yes & No \\
\hline $\begin{array}{l}\text { In vivo expression } \\
\text { of protein }\end{array}$ & $\begin{array}{l}\text { Optional: epitope-tagged } \\
\text { protein. }\end{array}$ & $\begin{array}{l}\text { Required: dam and } \\
\text { dam-fusion protein. }\end{array}$ \\
\hline Mapping resolution & $\begin{array}{l}0.5-3 \mathrm{~kb} \text {, depending on } \\
\text { fragment size. }\end{array}$ & $2 \mathrm{~kb}$ \\
\hline Time resolution & Minutes & Approximately 1 day. \\
\hline Possible artifacts & $\begin{array}{l}\text { Potentially biased by "cross- } \\
\text { linkability"; epitope may be } \\
\text { blocked. }\end{array}$ & $\begin{array}{l}\text { Dam-fusion protein } \\
\text { may not be functional. }\end{array}$ \\
\hline $\begin{array}{l}\text { Detection of post- } \\
\text { translational modifications }\end{array}$ & Yes & No \\
\hline Current application & Yeast and human cell lines. & Drosophila cell line. \\
\hline
\end{tabular}

presents a strong argument that DamID indeed identifies genuine targets. We have also performed the mapping of target loci of chromatin proteins that most likely do not contact DNA directly, such as HP1, dSir2, and Su(var)3-9 (F. Greil and B.v.S, unpublished data and References 18 and 52). These mapping efforts have thus far only employed conventional cDNA arrays, which only allow for the detection of protein binding within or close $(<2 \mathrm{~kb})$ to transcribed regions. Despite these limitations, a large number of target loci have been identified for each of these proteins by cDNA array detection. In the future, arrays of intergenic regions will broaden considerably the potential of the DamID technology. Attempts by several laboratories to apply DamID in various other organisms, such as plants, worms, and mammalian cells are in progress.

\section{COMPARISON OF ChIP TO DamID}

Because of their fundamentally different underlying principles, DamID and ChIP each have distinct advantages and drawbacks (Table 2). (i) ChIP identifies sequences directly bound to a nucleosome or protein complex so that it might achieve a resolution of several hundred base pairs, whereas DamID identifies flanking regions with less resolution. This also means that DamID can often be used with standard genebased microarrays where regulatory regions are a few kilobases away from a gene, whereas ChIP requires close proximity to a target sequence. (ii) DamID requires the construction of a fusion protein, which, in some cases, can cause functional defects in the protein of interest. Moreover, it is sometimes difficult to confirm with certainty that the chimeric protein behaves identically to the unfused protein. (iii) DamID cannot be used to detect posttranslational modifications, such as those on histones, although it is suited to monitor the binding of proteins that recognize specific histone modifications, such as HP1 $(18,63)$. (iv) In contrast to DamID, ChIP requires a highly specific antibody against the protein of interest. If such an antibody is unavailable, then in vivo expression of an epitope-tagged protein may be used, but this has some of the same drawbacks as the expression of a dam-fusion protein. (v) DamID does not have the caveats related to cross-linkability and epitope accessibility; differences in chromatin accessibility between loci are quantitatively accounted for by a dam-only reference experiment $(18,52)$. (vi) With 
DamID, it is not possible to monitor rapid changes in protein binding (e.g., in different phases of the cell cycle) because the current DamID protocol requires cells to express the dam-fusion protein for about one day before genomic DNA is isolated. ChIP does not have this disadvantage because formaldehyde cross-linking occurs within minutes. (vii) DamID has not yet been confirmed to work in mammalian cells, whereas ChIP-on-chip studies of human transcription factors have been published $(48,50,51)$.

A future side-by-side comparison of the two technologies applied to the same proteins may yield more insights into the strengths and weaknesses of each approach. In any case, DamID and ChIP can be expected to serve as two complementary technologies toward the same goal-gaining insight into epigenetic mechanisms.

\section{CONCLUSION}

Profiling methodologies for understanding the epigenome are still in their infancy, and we expect rapid progress. With broader availability and the lowering costs of microarrays, the types of experiments described here should become increasingly routine for researchers. Arrays that cover contiguous genomic segments are especially desirable for epigenomics, and we expect that the demand will increase as researchers become more familiar with the profiling technologies that we have described.

Current approaches to understanding genetic regulatory networks often attempt to draw conclusions based on common expression; however, knowledge of the binding sites for the transcription factors that regulate the genes is potentially a more direct route to understanding regulatory mechanisms. These sites can be determined using ChIP-on-chip and DamID. Bioinformatics tools will be essential to deduce the full biological meaning of these protein-binding patterns and other epigenomic data. Algorithms have already been developed and applied to identify putative regulatory sequence motifs and to begin to dissect the complex networks of protein-DNA interactions $(47,61)$. The rapid emergence of new computational approaches, together with the profiling technologies that we have discussed here, will undoubtedly hasten our understanding of epigenetic mechanisms.

\section{ACKNOWLEDGMENTS}

We thank members of our laboratories for helpful suggestions. B.v.S. is supported by the Dutch Cancer Society (KWF) and NWO-Genomics.

\section{REFERENCES}

1.Ideker, T., V. Thorsson, J.A. Ranish, R. Christmas, J. Buhler, J.K. Eng, R. Bumgarner, D.R. Goodlett, et al. 2001. Integrated genomic and proteomic analyses of a systematically perturbed metabolic network. Science 292:929-934.

2.DeRisi, J.L., V. Iyer, and P.O. Brown. 1997. Exploring the metabolic and genetic control of gene expression on a genomic scale. Science 278:680-686.

3.Henikoff, S. and M.A. Matzke. 1997. Exploring and explaining epigenetic effects. Trends Genet. 13:293-295.

4.Rakyan, V. and E. Whitelaw. 2003. Transgenerational epigenetic inheritance. Curr. Biol. 13:R6.

5.Fatemi, M., A. Hermann, S. Pradhan, and A. Jeltsch. 2001. The activity of the murine DNA methyltransferase Dnmt1 is controlled by interaction of the catalytic domain with the $\mathrm{N}$-terminal part of the enzyme leading to an allosteric activation of the enzyme after binding to methylated DNA. J. Mol. Biol. 309:11891199.

6.Leonhardt, H., A.W. Page, H.U. Weier, and T.H. Bestor. 1992. A targeting sequence directs DNA methyltransferase to sites of DNA replication in mammalian nuclei. Cell 71:865873

7.Turner, B.M. 2002. Cellular memory and the histone code. Cell 111:285-291.

8.Ahmad, K. and S. Henikoff. 2002. Epigenetic consequences of nucleosome dynamics. Cell 111:281-284.

9.Bird, A. 2002. DNA methylation patterns and epigenetic memory. Genes Dev. 16:6-21.

10.Jackson, J.P., A.M. Lindroth, X. Cao, and S.E. Jacobsen. 2002. Control of CpNpG DNA methylation by the KRYPTONITE histone H3 methyltransferase. Nature 416:556-560.

11. Tamaru, H. and E.U. Selker. 2001. A histone H3 methyltransferase controls DNA methylation in Neurospora crassa. Nature 414:277283.

12.Waterborg, J.H. 1993. Dynamic methylation of alfalfa histone H3. J. Biol. Chem. 268:49184921.

13.Zilberman, D., X. Cao, and S.E. Jacobsen. 2003. ARGONAUTE4 controls of locus-specific siRNA accumulation and DNA and histone methylation. Science 299:716-719.
14.Soppe, W.J., Z. Jasencakova, A. Houben, T. Kakutani, A. Meister, M.S. Huang, S.E. Jacobsen, I. Schubert, and P.F. Fransz. 2002. DNA methylation controls histone $\mathrm{H} 3$ lysine 9 methylation and heterochromatin assembly in Arabidopsis. EMBO J. 21:6549-6559.

15.Workman, J.L. and R.E. Kingston. 1998. Alteration of nucleosome structure as a mechanism of transcriptional regulation. Ann. Rev. Biochem. 67:545-579.

16.Iyer, V.R., C.E. Horak, C.S. Scafe, D. Botstein, M. Snyder, and P.O. Brown. 2001. Genomic binding sites of the yeast cell-cycle transcription factors $\mathrm{SBF}$ and MBF. Nature 409:533-538.

17.Ren, B., F. Robert, J.J. Wyrick, O. Aparicio, E.G. Jennings, I. Simon, J. Zeitlinger, J. Schreiber, et al. 2000. Genome-wide location and function of DNA binding proteins. Science 290:2306-2309.

18.van Steensel, B., J. Delrow, and S. Henikoff. 2001. Chromatin profiling using targeted DNA adenine methyltransferase. Nat. Genet. 27:304-308.

19.Yan, P.S., C.M. Chen, H. Shi, F. Rahmatpanah, S.H. Wei, C.W. Caldwell, and T.H. Huang. 2001. Dissecting complex epigenetic alterations in breast cancer using $\mathrm{CpG}$ island microarrays. Cancer Res. 61:8375-8380.

20.Tompa, R., C.M. McCallum, J. Delrow, J.G. Henikoff, B. van Steensel, and S. Henikoff. 2002. Genome-wide profiling of DNA methylation reveals transposon targets of CHROMOMETHYLASE3. Curr. Biol. 12:65-68.

21.Novik, K.L., I. Nimmrich, B. Genc, S. Maier, C. Piepenbrock, A. Olek, and S. Beck. 2002. Epigenomics: genome-wide study of methylation phenomena. Curr. Issues Mol. Biol. 4:111-128.

22.Hatada, I., A. Kato, S. Morita, Y. Obata, K. Nagaoka, A. Sakurada, M. Sato, A. Horii, et al. 2002. A microarray-based method for detecting methylated loci. J. Hum. Genet. 47:448-451.

23.Zweiger, G. and R.W. Scott. 1997. From expressed sequence tags to "epigenomics": an understanding of disease processes. Curr. Opin. Biotechnol, 8:684-687.

24.Bernstein, B.E., E.L. Humphrey, R.L. Erlich, R. Schneider, P. Bouman, J.S. Liu, T. Kouzarides, and S.L. Schreiber. 2002. Methylation of histone H3 Lys 4 in coding regions of active genes. Proc. Natl. Acad. Sci. USA 99:8695-8700.

25.Kurdistani, S.K., D. Robyr, S. Tavazoie, and M. Grunstein. 2002. Genome-wide binding map of the histone deacetylase Rpd3 in yeast. Nat. Genet. 31:248-254.

26.Plass, C. 2002. Cancer epigenomics. Hum. Mol. Genet. 11:2479-2488.

27.Lyko, F. 2001. DNA methylation learns to fly. Trends Genet. 17:169-172.

28.Rabinowicz, P.D., K. Schutz, N. Dedhia, C. Yordan, L.D. Parnell, L. Stein, W.R. McCombie, and R.A. Martienssen. 1999. Differential methylation of genes and retrotransposons facilitates shotgun sequencing of the maize genome. Nat. Genet. 23:305-308.

29.Razin, A. 1998. CpG methylation, chromatin structure and gene silencing - a three-way connection. EMBO J. 17:4905-4908.

30.Fuks, F., P.J. Hurd, D. Wolf, X. Nan, A.P. 
Bird, and T. Kouzarides. 2002. The methylCpG-binding protein MeCP2 links DNA methylation to histone methylation. J. Biol Chem. 278:4035-4040.

31.Bestor, T.H. 2000. The DNA methyltransferases of mammals. Hum. Mol. Genet 9:2395-2402.

32.Jones, P.A. and S.B. Baylin. 2002. The fundamental role of epigenetic events in cancer. Nat Rev. Genet. 3:415-428.

33.Fraga, M.F. and M. Esteller. 2002. DNA methylation: A profile of methods and applications. BioTechniques 33:632-649.

34.Frommer, M., L.E. McDonald, D.S. Millar, C.M. Collis, F. Watt, G.W. Grigg, P.L. Molloy, and C.L. Paul. 1992. A genomic sequencing protocol that yields a positive display of 5methylcytosine residues in individual DNA strands. Proc. Natl. Acad. Sci. USA 89:18271831

35.Toyota, M., C. Ho, N. Ahuja, K.W. Jair, Q. Li, M. Ohe-Toyota, S.B. Baylin, and J.P. Issa. 1999. Identification of differentially methylated sequences in colorectal cancer by methylated $\mathrm{CpG}$ island amplification. Cancer Res. 59:2307-2312.

36.Shiraishi, M., A. Sekiguchi, M.J. Terry, A.J. Oates, Y. Miyamoto, Y.H. Chuu, M. Munakata, and T. Sekiya. 2002. A comprehensive catalog of $\mathrm{CpG}$ islands methylated in human lung adenocarcinomas for the identification of tumor suppressor genes. Oncogene 21:3804-3813.

37.Wei, S.H., C.M. Chen, G. Strathdee, J. Harnsomburana, C.R. Shyu, F. Rahmatpanah, H. Shi, S.W. Ng, et al. 2002. Methylation microarray analysis of late-stage ovarian carcinomas distinguishes progression-free survival in patients and identifies candidate epigenetic markers. Clin. Cancer Res. 8:2246-2252.

38.Lindroth, A.M., X. Cao, J.P. Jackson, D. Zilberman, C.M. McCallum, S. Henikoff, and S.E. Jacobsen. 2001. Requirement of CHROMOMETHYLASE3 for maintenance of CpXpG methylation. Science 292:20772080.

39.Gitan, R.S., H. Shi, C.M. Chen, P.S. Yan, and T.H. Huang. 2002. Methylation-specific oligonucleotide microarray: a new potentia for high-throughput methylation analysis. Genome Res. 12:158-164.

40.Adorjan, P., J. Distler, E. Lipscher, F. Model, J. Muller, C. Pelet, A. Braun, A.R. Florl, et al. 2002. Tumour class prediction and discovery by microarray-based DNA methylation analysis. Nucleic Acids Res. 30:e21.

41.Gilbert, D.M. 2002. Replication timing and transcriptional control: beyond cause and effect. Curr. Opin. Cell Biol. 14:377-383.

42.Raghuraman, M.K., E.A. Winzeler, D. Collingwood, S. Hunt, L. Wodicka, A. Conway, D.J. Lockhart, R.W. Davis, et al. 2001. Replication dynamics of the yeast genome. Science 294:115-121.

43.Schübeler, D., D. Scalzo, C. Kooperberg, B. van Steensel, J. Delrow, and M. Groudine. 2002. Genome-wide DNA replication profile for Drosophila melanogaster: a link between transcription and replication timing. Nat. Genet. 32:438-442.

44.Orlando, V. 2000. Mapping chromosomal proteins in vivo by formaldehyde-crosslinked- chromatin immunoprecipitation. Trends Biochem. Sci. 25:99-104.

45.Solomon, M.J. and A. Varshavsky. 1985. Formaldehyde-mediated DNA-protein crosslinking: a probe for in vivo chromatin structures. Proc. Natl. Acad. Sci. USA 82:64706474.

46.Lieb, J.D., X. Liu, D. Botstein, and P.O. Brown. 2001. Promoter-specific binding of Rap1 revealed by genome-wide maps of protein-DNA association. Nat. Genet. 28:327334

47.Lee, T.I., N.J. Rinaldi, F. Robert, D.T. Odom, Z. Bar-Joseph, G.K. Gerber, N.M. Hannett, C.T. Harbison, et al. 2002. Transcriptional regulatory networks in Saccharomyces cerevisiae. Science 298:799-804.

48.Weinmann, A.S., P.S. Yan, M.J. Oberley, T.H. Huang, and P.J. Farnham. 2002. Isolating human transcription factor targets by coupling chromatin immunoprecipitation and $\mathrm{CpG}$ island microarray analysis. Genes Dev. 16:235-244.

49.Robyr, D., Y. Suka, I. Xenarios, S.K. Kurdistani, A. Wang, N. Suka, and M. Grunstein. 2002. Microarray deacetylation maps determine genome-wide functions for yeast histone deacetylases. Cell 109:437-446.

50.Wells, J., P.S. Yan, M. Cechvala, T. Huang, and P.J. Farnham. 2003. Identification of novel $\mathrm{pRb}$ binding sites using $\mathrm{CpG}$ microarrays suggests that $E 2 F$ recruits $p R b$ to specific genomic sites during $\mathrm{S}$ phase. Oncogene 22:1445-1460.

51.Ren, B., H. Cam, Y. Takahashi, T. Volkert, J. Terragni, R.A. Young, and B.D. Dynlacht. 2002. E2F integrates cell cycle progression with DNA repair, replication, and $\mathrm{G}(2) / \mathrm{M}$ checkpoints. Genes Dev. 16:245-256.

52.van Steensel, B. and S. Henikoff. 2000. Identification of in vivo DNA targets of chromatin proteins using tethered dam methyltransferase. Nat. Biotechnol. 18:424-428.

53.Barras, F. and M.G. Marinus. 1989. The great GATC: DNA methylation in E. coli. Trends Genet. 5:139-143.

54.Singh, J. and A.J. Klar. 1992. Active genes in budding yeast display enhanced in vivo accessibility to foreign DNA methylases: a novel in vivo probe for chromatin structure of yeast. Genes Dev. 6:186-196.

55.Boivin, A. and J.M. Dura. 1998. In vivo chromatin accessibility correlates with gene silencing in Drosophila. Genetics 150:1539-1549.

56.Wines, D.R., P.B. Talbert, D.V. Clark, and S. Henikoff. 1996. Introduction of a DNA methyltransferase into Drosophila to probe chromatin structure in vivo. Chromosoma 104:332-340.

57.Lebrun, E., G. Fourel, P.A. Defossez, and E. Gilson. 2003. A methyltransferase targeting assay reveals silencer-telomere interactions in budding yeast. Mol. Cell Biol. 23:1498-1508.

58.Kwoh, T.J., D.Y. Kwoh, A.W. McCue, G.R. Davis, D. Patrick, and T.R. Gingeras. 1986. Introduction and expression of the bacterial PaeR7 methylase gene in mammalian cells. Proc. Natl. Acad. Sci. USA 83:7713-7717.

59.Misteli, T. 2001. Protein dynamics: implications for nuclear architecture and gene expression. Science 291:843-847.

60.Urig, S., H. Gowher, A. Hermann, C. Beck,
M. Fatemi, A. Humeny, and A. Jeltsch. 2002. The Escherichia coli dam DNA methyltransferase modifies DNA in a highly processive reaction. J. Mol. Biol. 319:1085-1096.

61.van Steensel, B., J. Delrow, and H.J. Bussemaker. 2003. Genomewide analysis of Drosophila GAGA factor target genes reveals context-dependent DNA binding. Proc. Natl. Acad. Sci. USA 100:2580-2585.

62.Orian, A., B. van Steensel, J. Delrow, H.J. Bussemaker, L. Li, T. Sawado, E. Williams, L.W. Loo, S.M. Cowley, et al. 2003. Genomic binding by the Drosophila Myc, Max, $\mathrm{Mad} / \mathrm{Mnt}$ transcription factor network. Genes Dev. 17:1101-1114.

63.Li, Y., D.A. Kirschmann, and L.L. Wallrath. 2002. Does heterochromatin protein 1 always follow code? Proc. Natl. Acad. Sci. USA 99(Suppl 4):16462-16469.

Address correspondence to:

Steven Henikoff

Howard Hughes Medical Institute and Division of Basic Sciences

Fred Hutchinson Cancer Research Center

1100 Fairview Ave. North

Seattle, WA 98109, USA

e-mail:steveh@fhcrc.org 